\title{
Non-Recursively Constructible Recursive Families of Graphs *
}

\author{
Colleen Bouey \\ Department of Mathematics \\ Loyola Marymount College \\ Los Angeles, CA 90045, USA \\ cbouey@lion.lmu.edu

\section{Aaron Ostrander} \\ Dept of Math and Computer Science \\ Berry College \\ Mount Berry, GA 30149, USA \\ aaron. ostrander@vikings . berry . edu
}

\author{
Christina Graves ${ }^{\dagger}$ \\ Department of Mathematics \\ The University of Texas at Tyler \\ Tyler, TX 75799, USA \\ cgraves@uttyler.edu \\ Gregory Palma \\ Dept of Math and Computer Science \\ Northeastern State University \\ Tahlequah, OK 74464, USA \\ palma@nsuok.edu
}

Submitted: Oct 25, 2011; Accepted: Apr 4, 2012; Published: Apr 16, 2012

Mathematics Subject Classification: 05C31

\begin{abstract}
In a publication by Noy and Ribó, it was shown that recursively constructible families of graphs are recursive. The authors also conjecture that the converse holds; that is, recursive families are also recursively constructible. In this paper, we provide two specific counterexamples to this conjecture, which we then extend to an infinite family of counterexamples.
\end{abstract}

\section{Introduction}

The rank of a graph $G=(V, E)$ is $|V|-k(G)$ where $k(G)$ is the number of connected components of $G$; the Tutte polynomial of the same graph has the expansion

$$
T(G ; x, y)=\sum_{A \subseteq E}(x-1)^{r(E)-r(A)}(y-1)^{|A|-r(A)}
$$

where $r(A)$ is the rank of the spanning subgraph $(V, A)$. An in-depth discussion of the Tutte polynomial can be found in [2] and [4]. The Tutte polynomial is related to many

${ }^{*}$ Research was supported by the National Science Foundation (NSF DMS-1062740).

${ }^{\dagger}$ Corresponding author 
other graph polynomials including the chromatic polynomial, reliability polynomial, and rank polynomial [4]. We pay special attention to the rank polynomial here. The rank polynomial of a graph $G=(V, E)$ is defined by

$$
R(G ; x, y)=\sum_{A \subseteq E} x^{r(E)-r(A)} y^{|A|-r(A)} .
$$

The rank polynomial is very closely related to the Tutte polynomial and their relationship is given by

$$
R(G ; x, y)=T(G ; x+1, y+1) .
$$

A family of graphs $\left\{G_{n}\right\}_{n \geqslant 0}$ is said to be recursive if the Tutte polynomials (or the analogous rank polynomials) satisfy a linear homogeneous recurrence relation; in other words, the Tutte polynomials must satisfy

$$
T\left(G_{n} ; x, y\right)=\alpha_{1} T\left(G_{n-1} ; x, y\right)+\alpha_{2} T\left(G_{n-2} ; x, y\right)+\cdots+\alpha_{r} T\left(G_{n-r} ; x, y\right)
$$

for all $n \geqslant r$ where $\alpha_{i}$ 's are polynomials in $x$ and $y$ with integer coefficients independent of $n$. This definition was first given in [1], and all of the recursive graphs mentioned in [1] have a unique property; they are all formed by starting with an initial graph and applying a sequence of elementary operations to get from $G_{n-1}$ to $G_{n}$. In [7], the authors described families of graphs formed in this manner as recursively constructible. To formally define this idea, we first introduce the notation that $N_{G}(W)$ is the set of vertices in $G$ adjacent to some vertex in $W$, and the definition is then given below.

Definition 1. A family of graphs $\left\{G_{n}\right\}_{n \geqslant 0}$ is recursively constructible if there exists a positive integer $r$ and a labeled graph $M$ such that:

(a) $V\left(G_{0}\right)=W_{0}$ and $V\left(G_{n}\right)=V\left(G_{n-1}\right) \cup W_{n}$,

(b) $N_{G_{n}}\left(W_{n}\right) \subseteq W_{0} \cup\left(\bigcup_{i=0}^{r} W_{n-i}\right)$ for $n>r$,

(c) $E\left(G_{0}\right)=E_{0}$ and $E\left(G_{n}\right)=\left(E\left(G_{n-1}\right) \backslash S\right) \cup E_{n}$, where $S \subseteq \bigcup_{i=1}^{r} E_{n-i}$.

(d) The subgraph of $G_{n}$ induced by $\left(W_{0} \cup\left(\bigcup_{i=0}^{r} W_{n-i}\right)\right.$ equals $M$ for $n>r$.

The word "equals" in condition (d) does not just mean that both graphs have exactly the same vertices and exactly the same edges. We also need the condition that both graphs are labeled exactly the same. More specifically, the subgraph of $G_{n}$ that is induced by the vertex set $W_{n}$ is always the same labeled graph and $\left|W_{n}\right|$ is constant for all $n$.

The basic idea is that we can construct $G_{n}$ from $G_{n-1}$ by deleting some edges and then adding some new vertices and edges so that the new vertices are only adjacent to vertices in $W_{0}, W_{n-1}, \ldots, W_{n-r+1}$ or $W_{n-r}$. Also during each subsequent construction, the exact same elementary operations are performed. Because the same procedure is followed each time, a transfer matrix technique proves useful for calculating the rank or Tutte polynomial of $G_{n}$. Using this technique, the authors of [7] show that the rank polynomial of $G_{n}$ is then given by

$$
R\left(G_{n} ; x, y\right)=x^{r\left(G_{n}\right)} \mathbf{X}_{0}^{t} \cdot \Lambda^{n} \cdot \mathbf{1}
$$




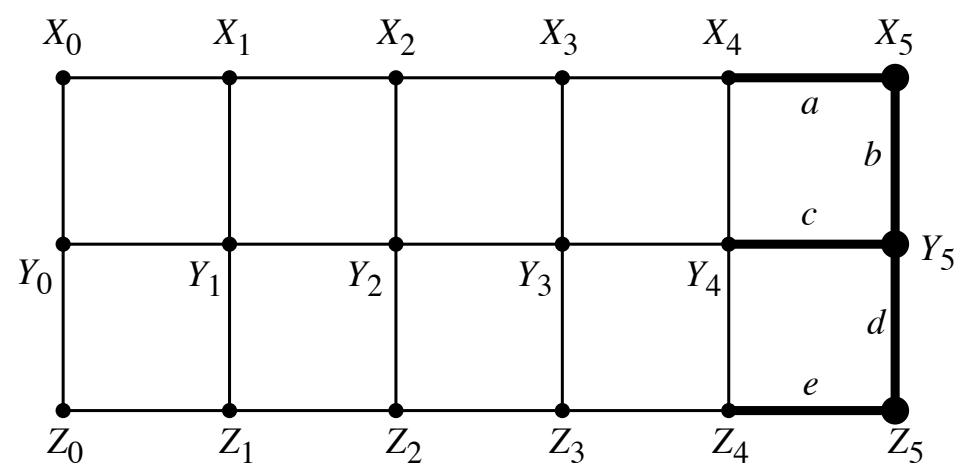

Figure 1: A double ladder graph.

where $\Lambda$ is the transfer matrix whose entries are rational functions in $x$ and $y$ and $\mathbf{X}_{0}$ is a vector that depends only on $G_{0}$. Because the family of graphs $\left\{G_{n}\right\}$ is recursively constructible, edges that are added in each step of the construction are incident to vertices in $W_{0} \cup\left(\bigcup_{i=1}^{r} W_{n-i}\right)$ in the previous step. If $m=\left|W_{0} \cup\left(\bigcup_{i=1}^{r} W_{n-i}\right)\right|$, the transfer matrix $\Lambda$ is a $B_{m} \times B_{m}$ matrix where $B_{m}$ is the Bell number of $m$, or the number of partitions of the set $W_{0} \cup\left(\bigcup_{i=1}^{r} W_{n-i}\right)$. It is worth noting that if $r=1$ and $W_{0}$ is not involved in the construction, then $m=\left|W_{n-1}\right|=\left|W_{n}\right|$. The columns and rows of $\Lambda$ are then labeled by these partitions. The entries of $\Lambda$ are explained in detail in [7] and for grid graphs in [3]. We give a brief example below.

For instance, consider the recursively constructible double ladder graph shown in Figure 1. The operations used to construct $G_{n}$ from $G_{n-1}$ are the addition of the five edges labeled $a, b, c, d$, and $e$ and the three vertices $X_{n}, Y_{n}$, and $Z_{n}$. These edges are incident to the vertices $X_{n-1}, Y_{n-1}$ and $Z_{n-1}$ in the graph $G_{n-1}$ and the vertices $X_{n}, Y_{n}$ and $Z_{n}$ in the graph $G_{n}$. The transfer matrix $\Lambda$ will be a $5 \times 5$ matrix with rows labels corresponding to the partitions of $\left\{X_{n-1}, Y_{n-1}, Z_{n-1}\right\}$ and column labels corresponding to the partitions of $\left\{X_{n}, Y_{n}, Z_{n}\right\}$. The first column will be labeled $\left\{X_{n}, Y_{n}, Z_{n}\right\}$; the second column $\left\{X_{n}, Y_{n}\right\},\left\{Z_{n}\right\}$; the third column $\left\{X_{n}, Z_{n}\right\},\left\{Y_{n}\right\}$; the fourth column $\left\{Y_{n}, Z_{n}\right\},\left\{X_{n}\right\}$; and the final column $\left\{X_{n}\right\},\left\{Y_{n}\right\},\left\{Z_{n}\right\}$.

The entries of $\Lambda$ are formed as follows. Let $B$ be a partition of $\left\{X_{n-1}, Y_{n-1}, Z_{n-1}\right\}$ and let $S$ be a subset of the edges $\{a, b, c, d, e\}$. We consider how the addition of the edges in $S$ affects the rank of a subgraph of $G_{n-1}$. For this, it is only necessary to know which of $\left\{X_{n-1}, Y_{n-1}, Z_{n-1}\right\}$ are in the same connected component. This is referred to as the state of the subgraph, which corresponds to a partition of $\left\{X_{n-1}, Y_{n-1}, Z_{n-1}\right\}$, and it is enough to study the effect of adding $S$ to each state. In our example, consider the subset $S=\{a, b\}$ and the partition $B=\left\{X_{n-1}, Y_{n-1}\right\},\left\{Z_{n-1}\right\}$; in other words, consider $X_{n-1}$ and $Y_{n-1}$ being in the same connected component and $Z_{n-1}$ being in a different component in some subgraph of $G_{n-1}$. By adding the two edges in $S$ to the subgraph of $G_{n-1}$, our new subgraph of $G_{n}$ will have $X_{n}$ and $Y_{n}$ in the same connected component and $Z_{n}$ in a different component. We will call this new state $B_{S}^{\prime}$. Let $\delta(B, S)$ be the change in the rank of $B$ produced by adding the edges $S$. For this particular $B$ and $S$, we have $\delta(B, S)=2$. Then, in the row labeled $B$ and the column labeled $B_{S}^{\prime}$, we add the term

$$
x^{-\delta(B, S)} y^{|S|-\delta(B, S)} .
$$


In this particular example, we would add the term $x^{-2} y^{0}$. We continue this process for every partition of the vertices $X_{n-1}, Y_{n-1}, Z_{n-1}$ and all subsets of the set of added edges. Each family of recursively constructible graphs also has an initial vector that corresponds to the contributions of the states from the initial graph. For the double ladder graph, the initial vector corresponds to the path of length two and is given by

$$
\left(\begin{array}{lllll}
x^{-2} & x^{-1} & 0 & x^{-1} & 1
\end{array}\right)^{t} .
$$

Besides describing transfer matrices for recursively constructible graphs, the main theorem presented in [7] is that recursively constructible graphs are recursive. The authors go on to conjecture that the converse is true up to Tutte equivalence. Two graphs are Tutte equivalent if they have the same Tutte polynomial. Formally, their conjecture is stated as follows.

Conjecture 2. If a family of graphs $\left\{G_{n}\right\}$ is recursive, then there exists a recursively constructible family of graphs $\left\{H_{n}\right\}$ so that $T\left(G_{n} ; x, y\right)=T\left(H_{n} ; x, y\right)$.

In the remainder of this paper we give some counterexamples to this conjecture and strengthen the hypotheses of this conjecture in order to exclude these counterexamples.

\section{The Counter-Examples}

The following definition provides us with the appropriate language for discussing our first counter-example of the conjecture.

Definition 3. Let $G_{1}=\left(V_{1}, E_{1}\right)$ and $G_{2}=\left(V_{2}, E_{2}\right)$ be disjoint graphs with $v_{1} \in V_{1}$ and $v_{2} \in V_{2}$. The one-point join of $G_{1}$ and $G_{2}$ with respect to $v_{1}$ and $v_{2}$, denoted $G_{1} \cdot G_{2}$ is the graph produced by merging the vertices $v_{1}$ and $v_{2}$ into a single vertex $v$ while preserving edge incidences.

It is well-known that $T\left(G_{1} \cdot G_{2} ; x, y\right)=T\left(G_{1} ; x, y\right) T\left(G_{2} ; x, y\right)$; however, in [6], it is also shown that any irreducible factors of the Tutte polynomial must arise from blocks. A graph is considered Tutte unique if every graph having the same Tutte polynomial is isomorphic to the graph itself. Thus, if a Tutte polynomial of a graph $G$ can be factored into irreducible terms so that each factor is the Tutte polynomial of a Tutte unique graph, then the graph $G$ is only Tutte equivalent to graphs that have the same blocks as itself.

As a first counter-example to the conjecture, we consider the following family of graphs. Let $G_{0}$ be a 3-cycle, $G_{1}$ a 4 -cycle, and $G_{2}$ the complete graph on four vertices. We also define $G$ to be a 3 -cycle. The rest of the family of graphs is constructed recursively so that $G_{n}=G_{n-3} \cdot G$ for all $n \geqslant 3$ (see Figure 2). We note that $G_{0}, G_{1}, G_{2}$ and $G$ are all Tutte unique. The graph $G_{n}$ has $\lceil(n-2) / 3\rceil$ blocks isomorphic to $G$ and one block isomorphic to $G_{i}$ for some $i=0,1$, or 2 . Thus, if $G_{n}$ is connected, it is Tutte equivalent only to other graphs made by one-point joining $\lceil(n-2) / 3\rceil$ copies of $G$ and one of $G_{0}, G_{1}$, or $G_{2}$. 

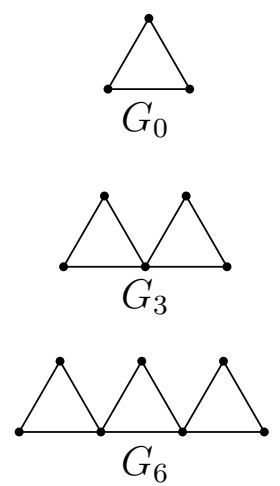

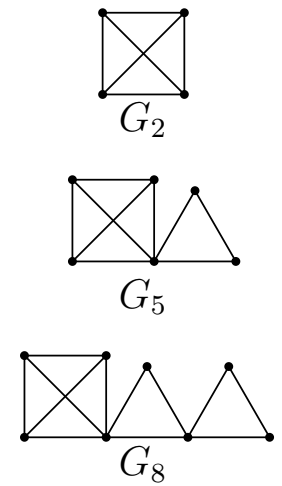

Figure 2: The family of graphs $\left\{G_{n}\right\}$ is not recursively constructible but is recursive.

We also notice that the Tutte polynomials of the family of graphs $\left\{G_{n}\right\}$ satisfy the following recurrence relation

$$
T\left(G_{n+3} ; x, y\right)=\left(x^{2}+x+y\right) T\left(G_{n} ; x, y\right)
$$

with Tutte polynomials for the initial graphs

$$
\begin{gathered}
T\left(G_{0}\right)=x^{2}+x+y, \quad T\left(G_{1}\right)=x^{3}+x^{2}+x+y, \\
T\left(G_{2}\right)=x^{3}+y^{3}+3 x^{2}+3 y^{2}+4 x y+2 x+2 y .
\end{gathered}
$$

Thus, the family of graphs $\left\{G_{n}\right\}$ is recursive. To see that this family is not recursively constructible notice that $\left|V\left(G_{4}\right)\right|-\left|V\left(G_{3}\right)\right|=1$ while $\left|V\left(G_{5}\right)\right|-\left|V\left(G_{4}\right)\right|=0$, thus there does not exist a set of vertices $W_{n}$ as required in part $(a)$ of Definition 1.

This same construction can be used to find a multitude of counter-examples. We will call families of graphs constructed in such a manner one-point periodically recursively constructible.

The one-point periodically recursively constructible families are not the only counterexamples to the conjecture of Noy and Ribó. The next counter-example we show arises from considering two important families of graphs. A prism graph, $Y_{n}$, is a graph on $2 n$ vertices that is the graph cartesian product of $K_{2}$ and the cycle graph $C_{n}$. The Mobius ladder graph, $M_{n}$, is a graph on $2 n$ vertices that is is the prism graph with a twist (see Figure 3).

In [1], the authors provide a recurrence relation obeyed by the Tutte polynomials of both the family $\left\{Y_{n}\right\}$ and $\left\{M_{n}\right\}$. This recurrence relation is the same for both families and is given by

$$
T\left(Y_{n} ; x, y\right)=\alpha_{1} T\left(Y_{n-1} ; x, y\right)+\cdots+\alpha_{6} T\left(Y_{n-6} ; x, y\right)
$$

and

$$
T\left(M_{n} ; x, y\right)=\alpha_{1} T\left(M_{n-1} ; x, y\right)+\cdots+\alpha_{6} T\left(M_{n-6} ; x, y\right)
$$

where $\alpha_{i}$ 's are polynomials in $x$ and $y$ independent of $n$. 




$H_{0}$

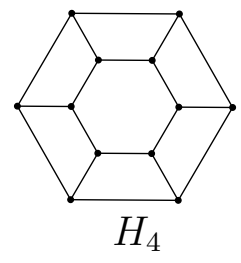

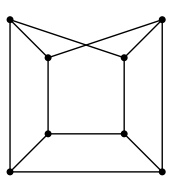

$H_{1}$



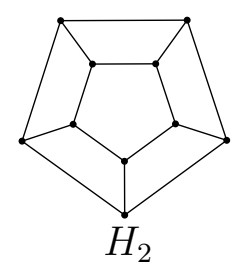
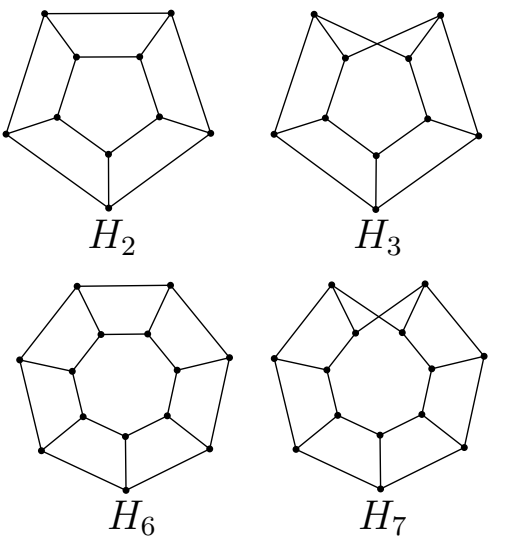

Figure 3: Graphs $H_{2 k}$ are the prism graphs $Y_{k+4}$. Graphs $H_{2 k+1}$ are the Mobius graphs $M_{k+4}$. The family of graphs $\left\{H_{n}\right\}$ is not recursively constructible but is recursive.

Consider the family of graphs $\left\{H_{n}\right\}$ where $H_{2 k}=Y_{k+4}$ and $H_{2 k+1}=M_{k+4}$ (see Figure 3 ). We see that the family of graphs $\left\{H_{n}\right\}_{n \geqslant 1}$ satisfies the recurrence relation

$$
T\left(H_{n} ; x, y\right)=\alpha_{1} T\left(H_{n-2} ; x, y\right)+\cdots+\alpha_{6} T\left(H_{n-12} ; x, y\right)
$$

where the $\alpha_{i}$ 's are the same as above.

This family is not recursively constructible because $\left|V\left(H_{1}\right)\right|-\left|V\left(H_{0}\right)\right|=0$ while $\left|V\left(H_{2}\right)\right|-\left|V\left(H_{1}\right)\right|=2$. The key characteristic of this counter-example is that the family of graphs $\left\{H_{2 k+1}\right\}_{k \geqslant 0}=\left\{M_{n}\right\}_{n \geqslant 4}$ and the family of graphs $\left\{H_{2 k}\right\}_{k \geqslant 0}=\left\{Y_{n}\right\}_{n \geqslant 4}$ have the exact same recurrence relation on their respective Tutte polynomials. Thus the recurrence relation on the Tutte polynomials for the entire family $\left\{H_{n}\right\}$ is very similar; it simply uses every other graph. This counter-example motivates the following definitions.

Definition 4. A family of graphs $\left\{H_{n}\right\}_{n \geqslant 0}$ is periodically recursively constructible if there exist recursively constructible families of graphs

$$
\left\{G_{0, n}\right\}_{n \geqslant 0},\left\{G_{1, n}\right\}_{n \geqslant 0}, \ldots,\left\{G_{m-1, n}\right\}_{n \geqslant 0},
$$

where $H_{r+q m}=G_{r, q}$ and there exists one labeled graph $M$ that satisfies the conditions in Definition 1 for all the families $\left\{G_{i, n}\right\}$. When $m$ is the least integer for which this holds, we say that $\left\{H_{n}\right\}$ is m-periodically recursively constructible.

Definition 5. A family of graphs $\left\{G_{n}\right\}$ is periodically recursive if the Tutte polynomials satisfy

$$
T\left(G_{n} ; x, y\right)=\alpha_{1} T\left(G_{n-m} ; x, y\right)+\alpha_{2} T\left(G_{n-2 m} ; x, y\right)+\cdots+\alpha_{r} T\left(G_{n-r m} ; x, y\right)
$$

for all $n \geqslant r m$ where $r$ and $m$ are positive integers and $\alpha_{i}$ 's are polynomials in $x$ and $y$ with integer coefficients independent of $n$. 
Recall that every recursively constructible family of graphs is recursive. From these definitions we see that a recursively constructible graph is 1-periodically recursively constructible and periodically recursive. We extend this result to all periodically recursively constructible families of graphs.

Theorem 6. Periodically recursively constructible families of graphs are periodically recursive.

Proof. Let $\left\{H_{n}\right\}_{n \geqslant 0}$ be an $m$-periodically recursively constructible family of graphs. Then there exist recursively constructible families of graphs

$$
\left\{G_{0, n}\right\}_{n \geqslant 0},\left\{G_{1, n}\right\}_{n \geqslant 0}, \ldots,\left\{G_{m-1, n}\right\}_{n \geqslant 0},
$$

where the sequence of operations to get from $G_{i, n}$ to $G_{i, n+1}$ is the same for all $i$, such that $H_{r+q m}=G_{r, q}$. In [7], the authors show that every recursively constructible family has a transfer matrix $\Lambda$ with entries that are rational in $x$ and $y$. Because all the families $\left\{G_{i, n}\right\}$ have the same $M$ from Definition 1, the rank generating function of the families $\left\{G_{i, n}\right\}$ is given by

$$
x^{r\left(G_{i}, 0\right)} \mathbf{X}_{\mathbf{i}, \mathbf{0}}^{\mathbf{t}}\left(I-x^{\left|W_{n}\right|} z \Lambda\right)^{-1} \mathbf{1}
$$

with $\Lambda$ and $\left|W_{n}\right|$ not depending on $i$. The expression $\left(I-x^{\left|W_{n}\right|} z \Lambda\right)^{-1}$ is a rational function, whereas $x^{r\left(G_{i}, 0\right)} \mathbf{X}_{\mathbf{i}, \mathbf{0}}^{\mathbf{t}}$ has only rational entries. Hence, the rank generating function is rational, and its denominator is given by $\left(I-x^{\left|W_{n}\right|} z \Lambda\right)^{-1}$ for all $i$. (There could potentially be some cancellations due to the term $x^{r\left(G_{i}, 0\right)} \mathbf{X}_{\mathbf{i}, \mathbf{0}}^{\mathbf{t}}$, but if so we do not perform them.) As the denominator of a rational generating function determines the recurrence relation satisfied by its coefficients, all the families $\left\{G_{i, n}\right\}_{n \geqslant 0}$ obey the same recurrence relation

$$
T\left(G_{k, n} ; x, y\right)=\alpha_{1} T\left(G_{k, n-1} ; x, y\right)+\alpha_{2} T\left(G_{k, n-2} ; x, y\right)+\cdots+\alpha_{p} T\left(G_{k, n-p} ; x, y\right)
$$

for all $k$ from 0 to $m-1$. Thus we have that

$$
\begin{aligned}
T\left(H_{k+m n} ; x, y\right) & =\alpha_{1} T\left(H_{k+m(n-1)} ; x, y\right)+\cdots+\alpha_{p} T\left(H_{k+m(n-p)} ; x, y\right) \\
& =\alpha_{1} T\left(H_{k+m n-m} ; x, y\right)+\cdots+\alpha_{p} T\left(H_{k+m n-p m} ; x, y\right)
\end{aligned}
$$

Since this holds for all $k$ from 0 to $m-1$ and $n \geqslant 0$ we see that $\left\{H_{n}\right\}_{n \geqslant 0}$ is periodically recursive.

Of course periodically recursive implies recursive, so as a corollary we have the following.

Corollary 7. Periodically recursively constructible families of graphs are recursive.

We could find many more examples of non-recursively constructible recursive families in this same manner. We note that the first counter-examples provided (using the onepoint joins) are in fact periodically recursively constructible as well. Given the above considerations we provide the following conjecture, a slightly modified version of the original conjecture of Noy and Ribó.

Conjecture 8. If a family of graphs $\left\{G_{n}\right\}$ is recursive but not periodically recursive with period greater than 1 , then there exists a recursively constructible family of graphs $\left\{H_{n}\right\}$ such that $T\left(G_{n} ; x, y\right)=T\left(H_{n} ; x, y\right)$. 


\section{Acknowledgements}

The authors would like the thank an anonymous referee for suggestions for the proof of Theorem 1.

\section{References}

[1] N. L. Biggs, R.M. Damerell, D.A. Sands, Recursive Families of Graphs, J. of Combinatorial Theory Ser. B 12 (1972) 123-131.

[2] T. Brylawski and J. Oxley, The Tutte polynomial and its application. Matroid applications, 123-225, Encyclopedia Math. Appl., 40, Cambridge Univ. Press, Cambridge, 1992.

[3] N. Calkin, C. Merino, S. Noble, M. Noy, Improve bounds for the number of forests and acyclic orientations in the square lattice, Electronic Journal of Combinatorics 10 (2003) \#R4.

[4] J.A. Ellis-Monaghan and C. Merino, Graph polynomials and their applications I: The Tutte polynomial, invited chapter for Structural Analysis of Complex Networks, Matthias Dehmer, ed., Birkhouser (2010).

[5] de Mier and Noy, On graphs determined by their Tutte polynomials, Graphs and Combinatorics 20 (2004) 105-119.

[6] C. Merino, A. de Mier and M. Noy, Irreducibility of the Tutte polynomial of a connected matroid, J. Combin. Theory Ser. B 83 (2001), 298-304.

[7] M. Noy, A. Ribó, Recursively constructible families of graphs, Advances in Appl. Mathematics 32 (2004) 350363 